\title{
Acute viral bronchiolitis: aetiology and treatment implications in a population that may be HIV co-infected
}

\author{
T Moodley, R Masekela, 0 Kitchin, S Risenga, RJ Green
}

T Moodley, R Masekela, 0 Kitchin, S Risenga, RJ Green, Division of Paediatric Pulmonology, Department of Paediatrics, University of Pretoria. E-mail: Robin.Green@up.ac.za

(P) Peer reviewed. (Submitted: 2009-11-04, Accepted: 2010-02-03). ๑ SAJEI

\section{Introduction}

Bronchiolitis is a disease where infection results in inflammation of the small airways and it occurs most commonly in children under 2 years of age. ${ }^{1}$ Most authors and experts agree that the condition has a viral aetiology. ${ }^{2}$ The commonest aetiology is respiratory syncitial virus (RSV) but parainfluenza virus (PIV) (especially type 3), influenza virus, adenovirus and some newer viruses have been identified. ${ }^{3}$ Despite the viral nature of this condition, and overwhelming evidence that bacterial co-infection is an extremely rare event, ${ }^{4,5}$ most general practitioners and even paediatricians in South Africa treat infants with bronchiolitis with an antibiotic. What is even more worrying is that the routine use of broad spectrum penicillins has made way for cephalosporins, macrolides and carbapenems. Recent guidelines have documented no additional benefit from routine antibiotic use in this condition. ${ }^{6,7}$ There are, however, some studies pointing to bacterial super-infection in children with severe bronchiolitis. ${ }^{8,9}$ One such study investigated 165 patients, with a median age of 1.6 months, and found that $70(42.2 \%)$ had a bronchoalveolar lavage culture positive for bacteria. In $36(21.8 \%)$, these were deemed to be co-infected, while $34(20.6 \%)$ had low bacterial growth and were deemed 'possibly' co-infected. All children were, however, ventilated for severe bronchiolitis and those co-infected required longer intensive care stay. White cell count (WCC) and C-reactive protein (CRP) did not differentiate between the groups and did not help to identify bacterial co-infection. ${ }^{8}$

Another trend that has crept into specialist practice is performing a CRP measurement on a blood sample of children with bronchiolitis. CRP measurement has been widely shown to be neither a sensitive nor a specific measure of bacterial disease in children with pneumonia. ${ }^{10-12}$ While most guidelines addressing pneumonia management in children suggest the routine use of antibiotics, those for bronchiolitis management discourage their use. Quite clearly failing to use clinical skills to differentiate between pneumonia and bronchiolitis contributes to poor treatment decision-making. Bronchiolitis is a disease of the peripheral airways and inflammation leads to air trapping with signs of hyperinflation such as the Hoover sign, loss of cardiac dullness and hepatoptosis. Pneumonia presents with fever, crepitations and signs of parenchymal consolidation. Some overlap of these conditions occurs but should then be defined clearly.

Bronchiolitis due to RSV is a disease of young infants in developed countries $^{13}$ but there is a suggestion of a later age of involvement in the developing world. The seasonality of bronchiolitis, although conventionally described as a winter disease, is not universally so in parts of South Africa. ${ }^{14}$ In KwaZulu-Natal there is a greater tendency
South Afr J Epidemiol Infect 2010;25(2):06-08

for bronchiolitis to occur throughout the year (Jeena P, personal communication). Risk factors for severe RSV-associated disease have been identified as gestational age less than 35 weeks at birth and chronological age less than 6 months, chronic lung disease in a child less than 2 years of age requiring medical therapy in the six months preceding admission for RSV bronchiolitis, children less than 2 years of age with cyanotic congenital heart disease or acyanotic congenital heart disease with a clinically significant shunt, and the presence of immunosuppression. ${ }^{14}$

The clinical diagnosis of viral pneumonia is made more frequently than that of bronchiolitis in HIV-infected children. ${ }^{15}$ An altered host immune response after RSV infection may explain the difference in clinical presentation observed between HIV-infected and uninfected children, with HIV-infected children having more severe disease and more frequent bronchopneumonic changes. Other differences observed in this group of patients include the risk for developing RSV-associated severe lower respiratory tract infection that persists beyond the first 6 months of life and that the incidence of concurrent bacteraemia is more common in HIV-infected children. ${ }^{15}$

Bronchiolitis is frequently incorrectly managed with antibiotic therapy. Despite the evidence that bacterial co-infection occurs in children with more severe disease, predicting this in mild or moderate disease is unclear. ${ }^{15}$

The aim of this study is to document the viral nature of bronchiolitis in a cohort of bronchiolitics in Pretoria by describing the microbiological profile of children with bronchiolitis at Steve Biko Academic Hospital, assessing the rate of bacterial co-infection in bronchiolitis and seeking laboratory (biochemical; immunological) parameters that predict mixed (both viral and bacterial) infection.

\section{Methods}

This was a prospective longitudinal study of all children (under 2 years of age) admitted to Steve Biko (Pretoria) Academic Hospital with bronchiolitis during a two-year period (January 2006 - December 2007). Virological and bacteriological parameters were assessed. In an attempt to define bacterial co-infection, blood culture, CRP and white blood cell count were measured. All samples were collected only at study entry and not sequentially.

Complete blood count was determined using the automated haematology analyser Advia 2120 (Siemens Diagnostics, South Africa). 
CRP was measured using an immunoturbidometric reaction (Beckman Coulter Synchron LX20 PRO, Beckman Coulter Incorporated, Fullerton, California, USA)

Blood cultures positive for growth were plated onto agar and sensitivity measured using a Kirby-Bauer technique (Bactec 9240, Becton Dickinson, Maryland, USA).

All children in this age group presenting to hospital with fast breathing, cough and noisy breathing in the period 1 January 2006 to 31 December 2007 were included. A chest radiograph was performed on every child. Children found to have pneumonic consolidation on chest X-ray (CXR) were excluded. These children were admitted to the paediatric shortstay ward. Immunofluorescence testing was done using Chemicon/ Millipore kit (Light Diagnostics) on nasopharyngeal aspirate samples for detection of respiratory viruses (parainfluenza 1,2 , and 3 , influenza $A$ and $\mathrm{B}$, cytomegalovirus, adenovirus and RSV).

HIV testing was done using fourth generation HIV ELISA assays, Architect (Abbott Diagnostics) and Modular E170 (Roche Diagnostics). HIV DNA was extracted from $100 \mu \mathrm{l}$ of whole blood using MagNa Pure LC DNA Isolation Kit III in a MagNa Pure instrument (Roche Diagnostics), then HIV polymerase chain reaction (PCR) was performed using an Amplicor HIV-1 DNA test, version 1.5 (Roche Diagnostics).

Children were managed by standard protocol which included oxygen by nasal prongs. No child was given an antibiotic.

\section{Results}

One hundred and six children with viral-proven bronchiolitis were identified in the two-year period. There were 52 males and 54 females with a male:female ratio of 1:1.04. The mean age for males was 6.2 months and for females 5.5 months.

The ages for children with bronchiolitis in whom RSV, parainfluenza virus, influenza virus and adenovirus were identified, are summarised in Table 1.There were five patients in whom two viruses were isolated.

Table 1: Summary statistics for age by viral isolate

\begin{tabular}{|l|c|c|c|}
\hline Viral isolate & $\begin{array}{c}\text { No. of positive } \\
\text { results }\end{array}$ & $\begin{array}{c}\text { Mean age in } \\
\text { months (SD) }\end{array}$ & $\begin{array}{c}95 \% \text { confidence } \\
\text { interval }\end{array}$ \\
\hline RSV & 62 & $4.81(4.28)$ & $(3.72 ; 5.89)$ \\
\hline Influenza virus & 9 & $6.67(3.97)$ & $(3.62 ; 9.72)$ \\
\hline Parainfluenza virus & 26 & $6.37(4.57)$ & $(4.52 ; 8.21)$ \\
\hline Adenovirus & 14 & $6.71(4.86)$ & $(3.91 ; 9.52)$ \\
\hline HIV & 14 & $8.07(3.85)$ & $(5.85 ; 10.30)$ \\
\hline
\end{tabular}

At Steve Biko Academic Hospital, bronchiolitis shows a winter predominance (Figure 2) related to RSV and parainfluenza virus infections in particular.

A positive bacterial blood culture was found in 18 patients (16.98\%). Fourteen of these were deemed to be contaminants as Staphylococcus epidermidis was isolated, and only four had a potential pathogen (one Streptococcus pneumoniae, one Klebsiella pneumoniae, one Escherichia coli and one Staphylococcus aureus). Among the patients tested only one was positive for both blood culture and CRP, while among the blood culture-negative patients 35 were CRP-positive, i.e. a very high false positive rate.

No correlation was found between CRP and white cell count (Figure 3) and between CRP and the neutrophil count (Figure 4) in either positive or negative blood culture groups.

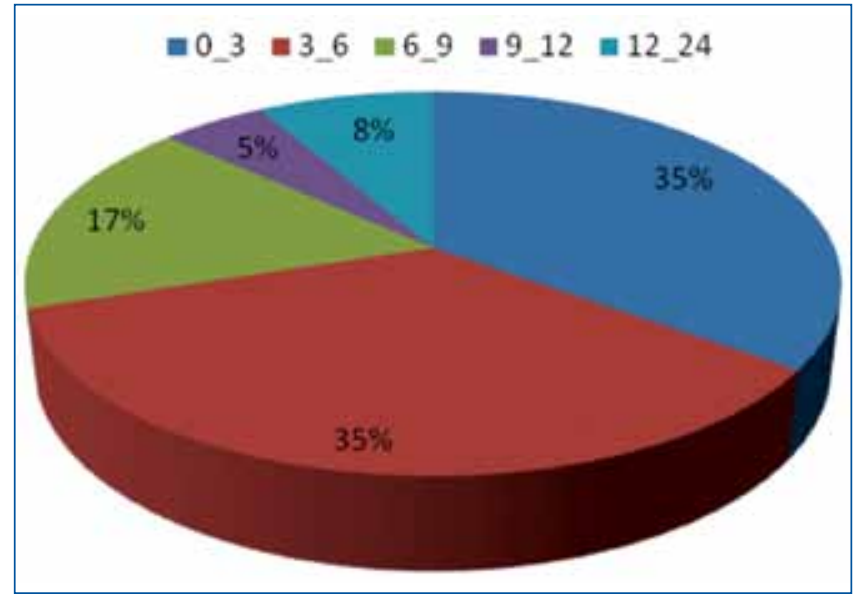

Figure 1: RSV age distribution (in months) for children with bronchiolitis at Steve Biko Academic Hospital (January 2006-December 2007)

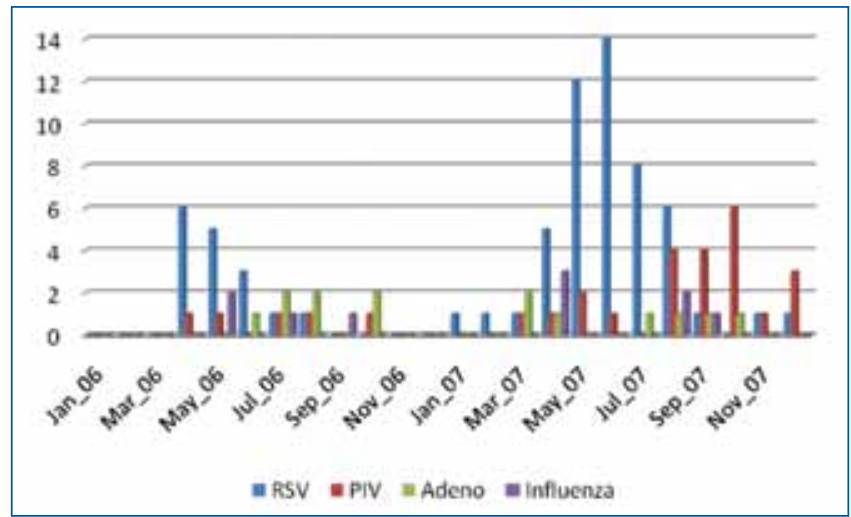

Figure 2: Number of viral isolates from children with bronchiolitis per month

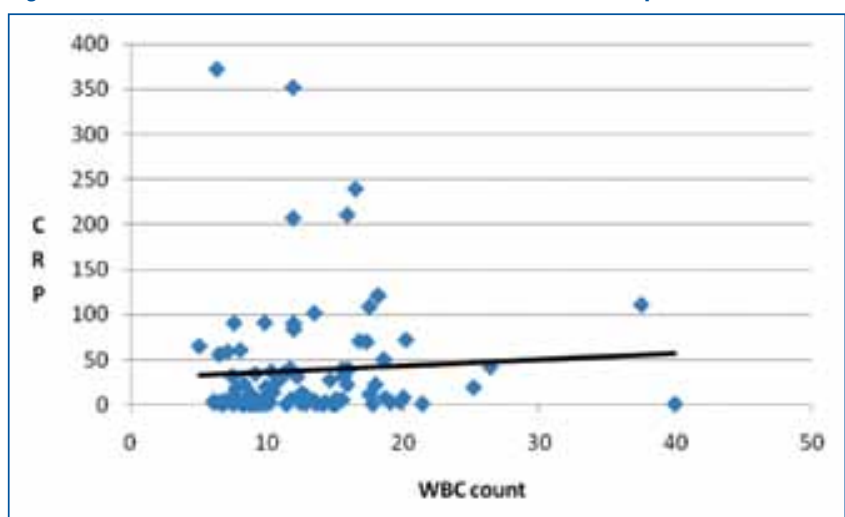

Figure 3: Correlation of CRP with white blood cell count in children with bronchiolitis, $R^{2}=0,0036$

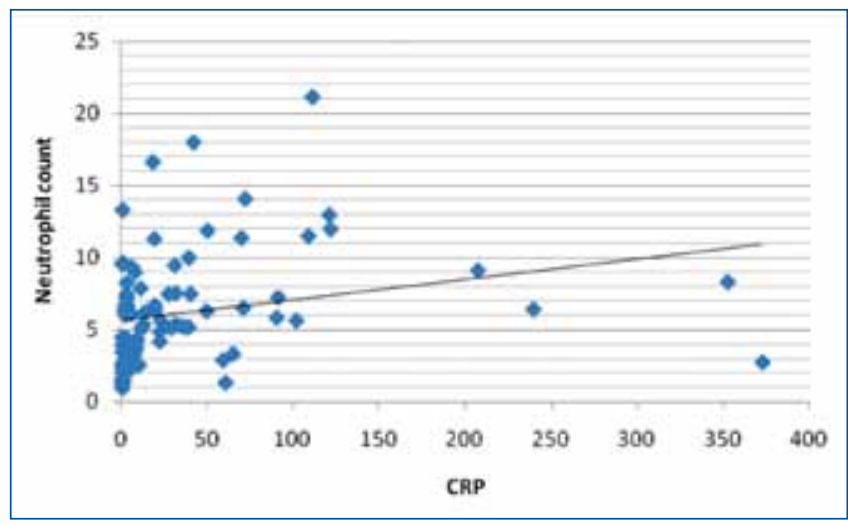

Figure 4: Correlation of neutrophil count with CRP in children with bronchiolitis, $R^{2}=0.055$ 
HIV was clinically suspected (presence of failure to thrive, hepatosplenomegaly, generalised lymphadenopathy or oral thrush) in 41 patients (38.7\%) and then proven (positive HIV ELISA and PCR) in 14 patients (13.2\%). The mean age of bronchiolitis in these children was 8 months, as opposed to the HIV-uninfected children where the mean age was 5.8 months. The HIV-infected bronchiolitics isolated the same viral organisms as the HIV-uninfected children, but the percentage composition was different (see Table 2).

Table 2: Proportion of positive viral isolates by HIV status

\begin{tabular}{|l|c|c|c|}
\hline Isolate & \multicolumn{2}{|c|}{ HIV } & Fisher's exact test \\
\hline & Positive & Negative & p value \\
\hline RSV & $33 \%(5 / 15)$ & $53.9 \%(14 / 26)$ & 0.330 \\
\hline Influenza virus & $6.67 \%(1 / 15)$ & $11.54 \%(3 / 26)$ & 1.000 \\
\hline Parainfluenza virus & $40 \%(6 / 15)$ & $26.9 \%(7 / 26)$ & 0.492 \\
\hline Adenovirus & $20 \%(3 / 15)$ & $11.54 \%(3 / 26)$ & 0.651 \\
\hline
\end{tabular}

Due to the wide scatter of CRP values, geometric means along with their $95 \%$ confidence intervals are reported comparing HIV-positive and -negative patients in terms of their CRP values (Table 3), $p$ value $=0.4736$.

Table 3: Summary statistics for CRP by HIV status

\begin{tabular}{|l|c|c|}
\hline HIV status & Geometric mean & 95\% confidence interval \\
\hline Positive & 18.44 & $(6.02 ; 56.43)$ \\
\hline Negative & 12.26 & $(6.49 ; 23.18)$ \\
\hline
\end{tabular}

\section{Discussion}

RSV bronchiolitis in children in Pretoria has a slightly older age distribution compared to that in developed countries. The mean age of bronchiolitis in HIV-infected children is higher than that of -uninfected children in keeping with the findings of earlier studies. ${ }^{3}$ Both HIVinfected and HIV-uninfected children had determinants of bacterial coinfection that did not correlate with one another. Neither white blood cell count, \% neutrophil count, CRP, nor blood culture of bacteria reliably suggest bacterial lung disease. We are unable to conclude from these variables what degree of bacterial pulmonary co-infection exists in viral bronchiolitis. However, none of the children in this study was treated with antibiotics and none suffered extended morbidity or mortality. We are therefore led to conclude that bacterial co-infection is both rare and inconsequential to true viral bronchiolitis. Since this study did not include children with a diagnosis of bronchopneumonia we limit our conclusions to bronchiolitis.

Since significant bacterial co-infection is rare in bronchiolitis, we recommend that the routine use of antibiotics for mild and moderately ill wheezy youngsters be discouraged. Bronchiolitis is a disease of viral origin (at least in our study of patients with mild to moderate disease) and whilst it is possible that bacterial co-infection occurs in children, especially those with more severe disease, the routine use of antibiotics is both inappropriate and potentially disastrous.

The under-representation of HIV-infected children in this study of bronchiolitis may have many explanations. Two of these may be that isolated viral bronchiolitis is uncommon in HIV-infected children and secondly that HIV infection confers a greater likelihood of more severe lung disease and bronchopneumonia. This study is unable to suggest a reason for this finding.

We suggest that there is an urgent need for South African bronchiolitis guidelines to manage this problem. Such guidelines must stress the differences between pneumonia (especially bronchopneumonia) and bronchiolitis. Routine antibiotic use is inappropriate in bronchiolitis. We would also encourage medical personnel to distinguish between bronchiolitis and bronchopneumonia as the inclusive diagnosis of bronchiolitis/bronchopneumonia in individual children is often interpreted as an excuse to use antibiotics. The routine use of bronchodilators and steroids, whilst a topic beyond the scope of this article, have also proven to be of limited value in this condition. ${ }^{6}$

Practitioners are urged to return to the evidence-based treatment of this very common condition. Misuse of antibiotics and CRP has both a cost, and also an implication for antimicrobial resistance. A number of limitations of this study are acknowledged. Firstly, the children treated for bronchiolitis were not followed clinically to determine long term morbidity and recurrence of disease and wheeze. It may be possible that bacterial co-infection may predict chronicity rather than severity of disease. Secondly, whilst this study attempted to test the value of the commonly employed diagnostic test, CRP, to predict bacterial disease, no attempt was made to determine if other diagnostic resources may have better discriminating ability. Such testing may have included serial CRP, procalcitonin (PCT) and cytokine assays (IL-1, 6). Again, although the evidence for the benefit of PCT is generally better than that of CRP, it is also not a useful screening test. ${ }^{16}$ The limited ability of blood culture to determine bacterial pneumonia is noted.

Infection in the lung is often difficult to prove. The diagnostic tests used in this study are the routine tests commonly used at this facility. It might be possible that both viral and bacterial organisms have not been detected by these laboratory methods.

\section{Conclusion}

Bronchiolitis remains a viral disease. In our study, CRP does not correlate with white cell count or bacterial blood culture. It is therefore, no more useful than those tests in predicting bacterial co-infection. Routine use of antibiotics should be discouraged since their use is no longer a benign intervention. Serious sequelae of both adverse events and emerging microbiological resistance, both in targeted organisms and those not targeted for therapy (collateral damage), are potential areas of concern.

Funding: The study was supported by an educational grant from Abbott Laboratories

\section{References}

Wohl MEB. In: Chernick V, Boat TF, Wilmott RW, Bush A, eds. Kendig's Disorders of the Respiratory Tract in Children, $7^{\text {th }}$ edition. Saunders: 2006

. Madhi SA, Venter M, Alexandra R, et al. Respiratory syncytial virus associated illness in high-risk children and national characterisation of the circulating virus genotype in South Africa. J Clinical Virol 2003; 27: 180-189 Morrow BM, Hatherill M, Smuts HE, Yeats J, Pitcher R, Argent AC. Clinical course of hospitalised children infected with human metapneumovirus and respiratory syncytial virus. J Paediatr Child Health 2006; 42(4): $174-178$

4. Greenes DS, Harper MB. Low risk of bacteremia in febrile children with recognizable viral syndromes. Pediatr Infect Dis J 1999: 18(3): 258-261

Purcell K, Fergie J. Concurrent serious bacterial infections in 2396 infants and children hospitalized with Purcell K, Fergie J. Concurrent serious bacterial infections in 2396 infants and children hospitalized with
respiratory syncytial virus lower respiratory tract infections. Arch Pediatr Adolesc Med 2002; 156(4): 322-324 Everard ML. Acute bronchiolitis and croup. Pediatr Clin N Am 2009; 56: 119-133

. American Academy of Pediatrics Subcomittee on Diagnosis and management of bronchiolitis. Diagnosis and management of bronchiolitis. Pediatrics 2006; 118: 1774-1793

8. Thorburn K, Harigopal S, Reddy V, Taylor N, Van Saene HKF. High incidence of pulmonary bacterial co-infection in children with severe respiratory syncitial virus (RSV) bronchiolitis. Thorax 2006; 61: 611-615

9. Madhi SA, Klugman KP. Madhi SA. A role for Streptococcus pneumoniae in virus-associated pneumonia. Nature Med 2004; 10:811-813

10. Gokhan T, Umit C, Derya A, et al Aetiological agents, interleukin-6, interleukin-8 and CRP concentrations in children with community- and hospital-acquired pneumonia. Annals Tropl Paediatr 2006; 26: 285-291

11. Seligman R. Decrease in procalcitonin and CRP are strong predictors of survival in ventilator-associated pneumonia. Crit Care 2006; 10: 125

12. Palmer A, Carlin JB, Freihorst $J$, et al. The use of CRP for diagnosing infections in young infants $<3$ months of age in developing countries. Annals Tropl Paediatr 2004; 24: 205-212

13. Venter M, Collinson M, Schoub BD. Molecular epidemiological analysis of community circulating respiratory syncytial virus in rural South Africa: Comparison of viruses and genotypes responsible for different disease manifestations. J Med Virol 2002: 68: 452-461

14. Madhi SA, Venter M, Alexandra $R$, et al Respiratory syncytial virus associated illness in high-risk children and national characterization of the circulating virus genotype in South Africa. J Clin Virol 2003; 27: 180-189

15. Madhi SA, Venter M, Madhi A, et al. Differing manifestations of respiratory syncytial virus-associated severe lower respiratory tract infections in human immunodeficiency virus type1-infected and uninfected children. Pediatr Infect Dis J 2001; 20: 164-170

17. Moulin $\mathrm{F}$, Raymond $\mathrm{J}$, et al. Procalcitonin in children admitted to hospital with community acquired pneumonia. Arch Dis Child 2001; 84: 332-336 